\title{
Maximum attainable field-free molecular orientation of a thermal ensemble with near-single-cycle THz pulses
}

\author{
Sheng-Lun Liao, ${ }^{1}$ Tak-San Ho, ${ }^{2}$ Herschel Rabitz, ${ }^{2}$ and Shih-I Chu ${ }^{1,3, *}$ \\ ${ }^{1}$ Center for Quantum Science and Engineering and Department of Physics, National Taiwan University, \\ Taipei 10617, Taiwan, Republic of China \\ ${ }^{2}$ Department of Chemistry, Princeton University, Princeton, New Jersey 08544, USA \\ ${ }^{3}$ Department of Chemistry, University of Kansas, Lawrence, Kansas 66045, USA
}

(Received 3 July 2012; published 25 January 2013)

\begin{abstract}
Recently, single-cycle $\mathrm{THz}$ pulses have been demonstrated in the laboratory to successfully induce field-free orientation in gas-phase polar molecules at room temperature [Phys. Rev. Lett. 107, 163603 (2011)]. In this paper, we examine the maximum attainable field-free molecular orientation with optimally shaped linearly polarized near-single-cycle THz laser pulses of a thermal ensemble. Large-scale benchmark optimal control simulations are performed, including rotational energy levels with the rotational quantum numbers up to $J=100$ for OCS linear molecules. The simulations are made possible by an extension of the recently formulated fast search algorithm, the two-point boundary-value quantum control paradigm, to the mixed-states optimal control problems in the present work. It is shown that a very high degree of field-free orientation can be achieved by strong, optimally shaped near-single-cycle THz pulses. The extensive numerical simulations showed that the maximum attainable $J$-dependent field-free orientation (equal to 0.714 for $J=60$ and 0.837 for $J=100$ at $100 \mathrm{~K}$ ) in the near-single-cycle $\mathrm{THz}$ pulse region is close to $92 \%$ of the corresponding optimal bound that can be attained by arbitrarily long pulses. It is also found that a smaller amplitude for the optimal control field corresponds to a smaller $J$ (e.g., $\approx 0.005$ a.u. for $J=60$ and $\approx 0.01$ a.u. for $J=100$ ) in the model simulations. The latter finding may underline the actual experimental performance of the field-free molecular orientation, since presently the available amplitude of single-cycle $\mathrm{THz}$ pulses can only reach slightly beyond $20 \mathrm{MV} / \mathrm{cm}$ $(\approx 0.0038$ a.u. $)$.
\end{abstract}

DOI: 10.1103/PhysRevA.87.013429

PACS number(s): $37.10 . \mathrm{Vz}, 32.80 . \mathrm{Qk}, 33.20 . \mathrm{Sn}, 42.50 . \mathrm{Hz}$

\section{INTRODUCTION}

Proper control of the alignment and orientation of molecules is important in many areas of physics and chemistry, including ultrafast dynamic imaging [1], molecular tomography [2], electron diffraction [3], chemical reactions [4,5], and high-harmonic generation [6,7]. The theories and techniques of molecular alignment have been well developed in the past ten years $[8,9]$. In particular, optimal control of molecular alignment not only helps to find the desired optimal control fields, but also minimizes the detrimental effect of dissipation due to the surrounding media [10]. The more challenging problem of orienting molecules is under intense development currently [11-19]. In general, high degrees of orientation can be achieved through either adiabatic or nonadiabatic processes. For example, excellent orientation for polar molecules can be achieved by a combination of dc and laser fields $[11,12]$. However, the presence of the dc field may be detrimental since it can alter the desired condition of performing experiments with field-free oriented molecules. It has also been suggested that orientation can be achieved by applying two-color laser field [13-15], half-cycle pulse [16,17], and few-cycle THz pulse [18] schemes. Only very recently, single-cycle $\mathrm{THz}$ pulses were used to attain field-free orientation in the laboratory [19].

Most field-free orientation schemes are based on the excitation of the rotational energy states of both odd and even rotational quantum numbers [20], in contrast to the

\footnotetext{
*sichu@ku.edu
}

prevalent schemes of aligning laser pulses that typically induce the excitation of the rotational energy states using primarily the second-order Stark dynamical couplings [21]. Theoretical studies have been performed on field-free orientation [22-24], and several monotonically convergent quantum control algorithms have been developed for finding optimal fields at both zero and finite temperatures [25-30]. For example, Sugny et al. proposed using a train of short pulses to control mixed-states quantum systems based on the sudden approximation [28]. A genetic algorithm $[25,26]$ has been utilized for identifying optimal control fields. However, very little theoretical work, especially in the context of optimal control theory, has explored the feasibility of molecular orientation in the single-cycle $\mathrm{THz}$ regime.

In this paper, we examine the attainable optimal degree of molecular orientation for a mixed-states thermal ensemble with linearly polarized near-single-cycle $\mathrm{THz}$ pulses at finite temperatures by extending a recently developed monotonically convergent optimal search algorithm-the twopoint boundary-value quantum control paradigm (TBQCP) [31-33] — within the framework of the density matrix formalism. The dc component of the control field is removed at each TBQCP search iteration with the aid of a bandpass filter [31]. An upper limit (optimal bound) for the degree of orientation can be identified, but it is difficult to know to what extent field-free orientation of a thermal molecular ensemble can be actually reached under various constraining conditions on the control fields. In particular, even though the orientation dynamics of a rigid rotor is controllable [28], the maximum attainable yield may still fall short of the ideal optimal bound 
when (i) the correction is included, for example, to account for the centrifugal distortion associated with large rotational quantum numbers, and (ii) the available single-cycle $\mathrm{THz}$ pulses are not only short, but also contain no dc component. A main goal of this work is to examine the achievable orientation with the currently available single-cycle linearly polarized $\mathrm{THz}$ laser pulses [34] by performing very large-scale benchmark optimal control simulations.

The remaining parts of the paper are arranged as follows. Section II describes the rotational motion of linear molecules and the optimal control bounds. Section III presents the mixed-states TBQCP that is to be used for finding optimal control fields in all numerical simulations in this paper. Section IV discusses and analyzes the optimal bounds of field-free orientation of OCS molecules (as nonrigid rotors) in near-single-cycle $\mathrm{THz}$ pulses. Finally, a short summary is given in Sec. $\mathrm{V}$.

\section{ORIENTATION CONTROL OF LINEAR MOLECULES AND OPTIMAL BOUNDS}

The time-dependent Hamiltonian $H(t)$ of a linear molecule in the presence of a control field $\mathcal{E}(t)$ is

$$
H(t)=H_{0}-\vec{\mu}(t) \cdot \overrightarrow{\mathcal{E}}(t),
$$

where $H_{0}$ is the field-free Hamiltonian and $\vec{\mu}(t)$ is the molecular dipole moment vector. Considering the controlled linear dipolar molecule as a nonrigid rotor [35], the corresponding field-free Hamiltonian is a diagonal matrix containing the energy levels denoted as $\varepsilon_{j}=B j(j+1)-D j^{2}(j+1)^{2}$, where $B$ is the rotational constant, $D$ is the centrifugal distortion constant, and $j$ is the rotational quantum number. In a linearly polarized control field, the coupling between the linear molecule and the control field can be denoted as $-\vec{\mu}(t) \cdot \overrightarrow{\mathcal{E}}(t)=-\mu_{0} \mathcal{E}(t) \cos \theta$, where $\theta$ is the polar angle between the molecular axis and the polarization vector of the laser field $\overrightarrow{\mathcal{E}}(t)$ and $\mu_{0}$ is the permanent dipole moment.

At temperature $\mathcal{T}$, the evolution of a molecular ensemble is described by the time-dependent equation for the density matrix $\rho(t)$, i.e.,

$$
\frac{\partial}{\partial t} \rho(t)=\frac{1}{\imath \hbar}[H(t), \rho(t)],
$$

subject to the initial condition

$$
\rho(0)=\sum_{j=0}^{\infty} \sum_{m=-j}^{j} \omega_{j}|j m\rangle\langle j m|,
$$

where $\quad \omega_{j}=\exp \left(-\varepsilon_{j} / k_{\mathrm{B}} \mathcal{T}\right) / \sum_{j}\left[(2 j+1) \exp \left(-\varepsilon_{j} / k_{\mathrm{B}} \mathcal{T}\right)\right]$ denotes the Boltzmann distribution of the mixed states in the thermal equilibrium, with $|j m\rangle$ being the rotational eigenenergy states associated with the rotational energy $\varepsilon_{j}$, which is independent of the magnetic quantum number $m$, and $k_{\mathrm{B}}$ is the Boltzmann constant.

The molecular orientation can be defined in terms of the observable operator $A=\cos \theta$, and the time-dependent orientation $\mathcal{A}(t)=\langle\langle\cos \theta\rangle\rangle(t)$ is the expectation values of $A$ at the time $t$, which may be expressed as

$$
\mathcal{A}(t)=\operatorname{Tr}\{A \rho(t)\}=\operatorname{Tr}\left\{\cos \theta U(t, 0) \rho(0) U^{\dagger}(t, 0)\right\},
$$

where $U(t, 0)$ is the time evolution operator associated with the control field $\mathcal{E}(t)$. In the following discussion, $J$ denotes the largest rotational quantum number, i.e., $0 \leqslant j \leqslant J$ in the numerical calculations, and $(J+1)^{2}$ is the total number of rotational energy levels.

For arbitrarily polarized laser fields, the time-dependent orientation $\mathcal{A}(t)$ for a chosen $J$ is bounded by the relation [28,36-38]

$$
\mathcal{A}(t) \leqslant\langle\langle A\rangle\rangle_{\mathrm{kin}}=\sum_{j=0}^{J} \sum_{m=-j}^{j} \omega_{j} \lambda_{j^{2}+j+m},
$$

where the $\omega_{i}$ 's and $\lambda_{i}$ 's are, respectively, the eigenvalues of $\rho(0)$ [see Eq. (3)] and $A$ in descending order, i.e., $\omega_{0} \geqslant \omega_{1} \geqslant$ $\cdots \omega_{(J+1)^{2}-1} \geqslant 0$ and $1>\lambda_{0} \geqslant \lambda_{1} \geqslant \cdots \geqslant \lambda_{(J+1)^{2}-1}>-1$, with $(J+1)^{2}$ being the number of eigenvalues of the matrix $A$. Note that $A$ has a zero trace, i.e., $\sum_{j=0}^{J} \sum_{m=-j}^{j} \lambda_{j^{2}+j+m}=0$, and $\lambda_{i}=-\lambda_{(J+1)^{2}-1-i}, i=0, \ldots,(J+1)^{2}-1$. From Eq. (5), it can then be shown that, as $J \rightarrow \infty$, the kinematic bound satisfies

$$
\lim _{J \rightarrow \infty}\langle\langle A\rangle\rangle_{\text {kin }} \rightarrow \sum_{j=0}^{\infty}(2 j+1) \omega_{j}=1,
$$

independent of temperature, since the distribution of $\lambda$ 's becomes infinitely dense around 1 asymptotically.

A linearly polarized laser field will not couple states with different magnetic quantum numbers [28]. As a result, the orientation problem can be decomposed into subspaces with each labeled by the magnetic quantum number $m=$ $-J, \ldots, J$. In particular, the density matrix $\rho(t)$, the evolution operator $U(t, 0)$, and the orientation operator $A=\cos \theta$ can be, respectively, cast in block matrix direct sums as follows: $\rho(t)=\bigoplus_{m=-J}^{J} \rho^{m}(t), U(t, 0)=\bigoplus_{m=-J}^{J} U^{m}(t, 0)$, and $A=$ $\bigoplus_{m=-J}^{J} A^{m}$, where the subblocks $\rho^{m}(t), U^{m}(t, 0)$, and $A^{m}$ are $(J-|m|+1) \times(J-|m|+1)$ square matrices defined on $2 J+1$ decoupled subspaces, each (herein $m$ subspace) spanned by $(J-|m|+1)$ angular momentum eigenstates $|j m\rangle$ with $j=|m|,|m|+1, \ldots, J$. Thus, Eq. (4) can be expanded as

$$
\begin{aligned}
\mathcal{A}(t) & =\sum_{m=-J}^{J} \sum_{j \geqslant|m|} \omega_{j}\left\langle j m\left|U^{m \dagger}(t, 0) A^{m} U^{m}(t, 0)\right| j m\right\rangle \\
& =\sum_{m=-J}^{J} \sum_{j \geqslant|m|} \sum_{j^{\prime} \geqslant|m|} \omega_{j} \lambda_{j^{\prime}}^{m}\left|\left\langle\lambda_{j^{\prime}}^{m}\left|U^{m}(t, 0)\right| j m\right\rangle\right|^{2},
\end{aligned}
$$

where $\left|\lambda_{j^{\prime}}^{m}\right\rangle$ is the $j^{\prime}$ th eigenstate of $A^{m}$ associated with the eigenvalue $\lambda_{j^{\prime}}^{m}$ such that $A^{m}\left|\lambda_{j^{\prime}}^{m}\right\rangle=\lambda_{j^{\prime}}^{m}\left|\lambda_{j^{\prime}}^{m}\right\rangle$. Note that $A^{m}$ is traceless, i.e., $\sum_{j=|m|}^{J} \lambda_{j}^{m}=0$. From Eq. (6), it can be shown that the time-dependent orientation $\mathcal{A}(t)$ for linearly polarized control fields is bounded by the relation

$$
\mathcal{A}(t) \leqslant\langle\langle A\rangle\rangle_{\text {lin }}=\sum_{m=-J}^{J} \sum_{j=|m|}^{J} \omega_{j} \lambda_{j}^{m},
$$

where $1>\lambda_{|m|}^{m}>\lambda_{|m|+1}^{m}>\cdots>\lambda_{J}^{m}>-1, m=0, \pm 1, \ldots$, $\pm J$, arranged in descending order within each $m$ subspace. 
The upper bound $\langle\langle A\rangle\rangle_{\text {lin }}$ is obtained when the condition

$$
\left|\left\langle\lambda_{j^{\prime}}^{m}\left|U^{m}(T, 0)\right| j m\right\rangle\right|^{2}=\left|\sum_{j^{\prime \prime} \geqslant|m|}\left\langle\lambda_{j^{\prime}}^{m} \mid j^{\prime \prime} m\right\rangle c_{j^{\prime \prime} m}^{j m}(T)\right|^{2}=\delta_{j j^{\prime}}
$$

is satisfied, where

$$
c_{j^{\prime} m}^{j m}(T)=\left\langle j^{\prime} m\left|U^{m}(T, 0)\right| j m\right\rangle
$$

denotes the transition probability amplitude to the final rotational energy state $\left|j^{\prime} m\right\rangle$ at the target time $T$, after evolving from the initial rotational energy state $|j m\rangle$ at $t=0$ within the $m$ subspace. Equation (8) shows that the upper bound $\langle\langle A\rangle\rangle_{\operatorname{lin}}$ is reached when every rotational energy level $|j m\rangle$ in the initial density matrix $\rho(0)$ evolves into a well-oriented wave packet perfectly aligned with the corresponding eigenstate $\left|\lambda_{j}^{m}\right\rangle$ of the orientation operator $A^{m}$, i.e., $U^{m}(T, 0)|j m\rangle=\exp (i \varphi)\left|\lambda_{j}^{m}\right\rangle$ (within an undefined phase $\varphi$ ).

From Eqs. (5) and (7), it is obvious that both the magnitudes of $\langle\langle A\rangle\rangle_{\text {kin }}$ and $\langle\langle A\rangle\rangle_{\text {lin }}$ increase with $J$ and ultimately converge to unity as $J \rightarrow \infty$. Furthermore, the ordered set

$$
\mathcal{S}_{1} \equiv\left\{\lambda_{0} \geqslant \lambda_{1} \geqslant \cdots \geqslant \lambda_{(J+1)^{2}-1}\right\}
$$

is equal to the partially ordered set

$\mathcal{S}_{2} \equiv\left\{\lambda_{0}^{0}>\lambda_{1}^{0}>\cdots>\lambda_{J}^{0} ; \lambda_{1}^{ \pm 1}>\lambda_{2}^{ \pm 1}>\cdots>\lambda_{J}^{ \pm 1} ; \ldots ; \lambda_{J}^{ \pm J}\right\}$,

both containing $(J+1)^{2}$ eigenvalues of the operator $A$. We note that (i) $\lambda_{j}^{m}=-\lambda_{J+|m|-j}^{m}, j=|m|, \ldots, J$; (ii) $\lambda_{j}^{m}=\lambda_{j}^{-m}$, $j \geqslant m=1, \ldots, J$; and (iii) $\lambda_{J-(J-|m|) / 2}^{m}=0$ whenever $J-$ $|m|+1$ is an odd integer. By comparing Eqs. (5) and (7), it is readily seen that for the same $J$ value,

$$
\langle\langle A\rangle\rangle_{\text {lin }} \leqslant\langle\langle A\rangle\rangle_{\text {kin }}
$$

in which the equality holds for all $J$ values when $\mathcal{T}=0$ and for $J \leqslant 2$ when $\mathcal{T}>0 \mathrm{~K}$.

In general, it is difficult to show to what extent the ideal optimal orientation bounds can be actually reached under various constraining conditions on the control laser fields. In particular, the issue of controllability using a single linearly polarized laser $[28,37]$ must be taken into account when the calculated optimal yield is smaller than the ideal optimal bound. In the next section, we describe a fast monotonically convergent searching algorithm (the TBQCP method [31]) in the context of a mixed-states density matrix for finding optimal control fields and examining the optimal bounds which are numerically achievable in the presence of a near-single-cycle linearly polarized $\mathrm{THz}$ laser.

\section{A MIXED-STATES TWO-POINT BOUNDARY-VALUE QUANTUM CONTROL PARADIGM}

Consider the time-dependent equation for the density matrix $\rho(t)$ of a quantum system interacting with a linearly polarized control field,

$$
\frac{\partial}{\partial t} \rho(t)=\frac{1}{\imath \hbar}\left[H_{0}-\mu_{0} \mathcal{E}(t) \cos \theta, \rho(t)\right],
$$

where $H_{0}$ is the field-free Hamiltonian, $\mu_{0}$ is the permanent dipole moment, and $\theta$ is the polar angle between the molecular axis and the polarization vector of the laser field $\mathcal{E}(t)$. The time evolution operator $U(t, 0)$, associated with the control field $\mathcal{E}(t)$, satisfies the following Schrödinger equation:

$$
\frac{\partial}{\partial t} U(t, 0)=\frac{1}{\imath \hbar}\left\{H_{0}-\mu_{0} \mathcal{E}(t) \cos \theta\right\} U(t, 0), U(0,0)=\mathbf{I} .
$$

In the presence of a linearly polarized laser, both the evolution operator $\hat{U}$ and the time-dependent density matrix $\hat{\rho}$, respectively, can be further expressed in block-diagonal matrices as

$\hat{U}(t, 0)=\left(\begin{array}{cccc}\hat{U}^{m=J}(t, 0) & 0 & \cdots & 0 \\ 0 & \hat{U}^{m=J-1}(t, 0) & \cdots & 0 \\ \vdots & \vdots & \ddots & \vdots \\ 0 & 0 & \cdots & \hat{U}^{m=-J}(t, 0)\end{array}\right)$

and

$$
\hat{\rho}(t)=\left(\begin{array}{cccc}
\hat{\rho}^{m=J}(t) & 0 & \cdots & 0 \\
0 & \hat{\rho}^{m=J-1}(t) & \cdots & 0 \\
\vdots & \vdots & \ddots & \vdots \\
0 & 0 & \cdots & \hat{\rho}^{m=-J}(t)
\end{array}\right),
$$

both containing uncoupled subblock matrices for different $m$ values.

The goal of quantum optimal control algorithms is to quickly find a control field $\mathcal{E}(t), t \in[0, T]$, that can steer the quantum system from an initial state $\rho(0)$ to a final state $\rho(T)$ in a finite time $T$ such that the expectation value $\langle\langle O(T)\rangle\rangle \equiv \operatorname{Tr}\{O \rho(T)\}$ of the target observable $O$ (for example, $O \equiv \cos \theta$ for molecular orientation control) is as close to its admissible maximum value as possible.

To formulate a monotonically convergent algorithm for obtaining optimal control fields by extending a recently formulated fast monotonically convergent optimal control search algorithm-the two-point boundary-value quantum control paradigm (TBQCP) [31-33]-to mixed-states quantum optimal control problems, we introduce a timedependent Hermitian operator $O(t)$ satisfying the invariant equation

$\frac{d}{d t} O(t) \equiv \frac{\partial}{\partial t} O(t)-\frac{1}{\imath \hbar}\left[H_{0}-\mu_{0} \mathcal{E}^{(0)}(t) \cos \theta, O(t)\right] O(t)=0$,

with the boundary condition $O(T)=O$ (which is the target observable), associated with a reference control field $\mathcal{E}^{(0)}(t)$. From Eq. (15), it can then be derived that the expectation $\operatorname{Tr}\left\{O(t) \rho^{(0)}(t)\right\}$ is independent of time $t$, where the density matrix $\rho^{(0)}(t)$ is also associated with the reference control field $\mathcal{E}^{(0)}(t)$ and satisfies the equation

$$
\frac{\partial}{\partial t} \rho^{(0)}(t)=\frac{1}{\imath \hbar}\left[H_{0}-\mu_{0} \mathcal{E}^{(0)}(t) \cos \theta, \rho^{(0)}(t)\right] .
$$

From Eqs. (11) and (15), we derive the relation

$$
\frac{d}{d t}\langle\langle O(t)\rangle\rangle \equiv \frac{d}{d t} \operatorname{Tr}\{O(t) \rho(t)\}=f_{\rho}(t)\left\{\mathcal{E}(t)-\mathcal{E}^{(0)}(t)\right\},
$$

where

$$
\langle\langle O(t)\rangle\rangle \equiv \operatorname{Tr}\{O(t) \rho(t)\}
$$


and

$$
f_{\rho}(t)=-\frac{1}{i \hbar} \operatorname{Tr}\left\{\left[O(t), \mu_{0} \cos \theta\right] \rho(t)\right\} .
$$

Integrating Eq. (17) leads to

$$
\langle\langle O(T)\rangle\rangle-\langle\langle O(0)\rangle\rangle=\int_{0}^{T} f_{\rho}(t)\left\{\mathcal{E}(t)-\mathcal{E}^{(0)}(t)\right\} d t,
$$

which constitutes a nonlinear integral equation for the control field $\mathcal{E}(t)$.

From Eqs. (17) and (20), a monotonically convergent iteration scheme can be readily obtained by choosing the control field $\mathcal{E}(t)$ according to the relation

$$
\mathcal{E}(t)=\mathcal{E}^{(0)}(t)+\eta S(t) f_{\rho}(t)
$$

where $\eta(>0)$ is a positive constant and $S(t)(\geqslant 0)$ is the shape function of the control field. It is then seen that substituting Eq. (21) into Eqs. (17) and (20) gives, respectively,

$$
\frac{d}{d t}\langle\langle O(t)\rangle\rangle=\eta S(t) f_{\rho}^{2}(t) \geqslant 0
$$

and

$$
\langle\langle O(T)\rangle\rangle-\langle\langle O(0)\rangle\rangle=\eta \int_{0}^{T} S(t)\left[f_{\rho}(t)\right]^{2} d t \geqslant 0,
$$

i.e., $\quad\langle\langle O(T)\rangle\rangle=\operatorname{Tr}\{O \rho(T)\} \geqslant \operatorname{Tr}\left\{O \rho^{(0)}(T)\right\}=\langle\langle O(0)\rangle$. Equation (21) forms a recurrence relation for the mixed-states two-point boundary-value quantum optimal paradigm (MS-TBQCP) that can be applied iteratively for refining the control field such that the targeted expectation $\langle\langle O(T)\rangle\rangle$ increases monotonically not only at each instant of time, but also throughout the iterations. All optimal control simulations in the following section (Sec. IV) have been carried out using the MS-TBQCP method presented above.

\section{RESULTS AND DISCUSSIONS}

The linear molecule OCS is used as an example to investigate how the temperature affects field-free orientation control by means of near-single-cycle $\mathrm{THz}$ optimal control laser pulses. The OCS molecule has a rotational constant

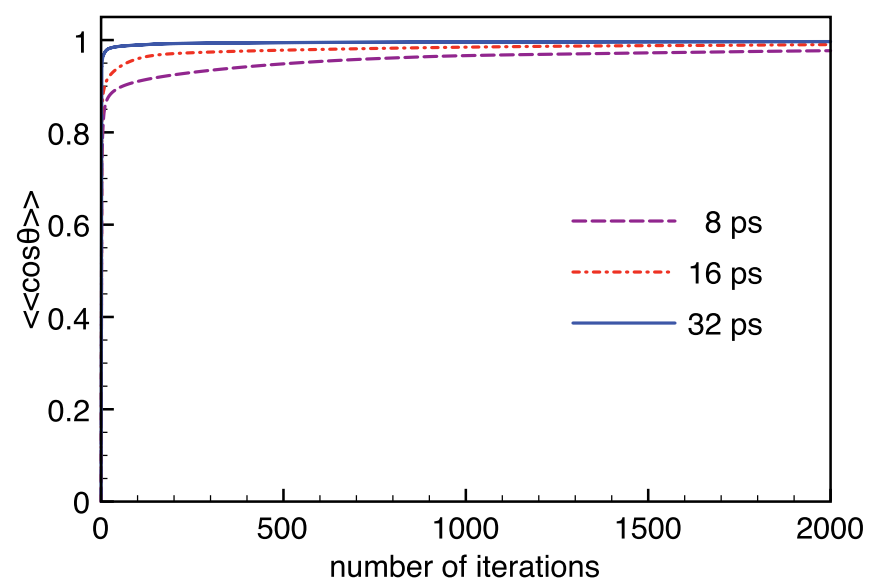

FIG. 1. (Color online) Convergent behavior of the OCS orientation at $J=60$ and $\mathcal{T}=0 \mathrm{~K}$ for different control pulse lengths: $T_{1}=8,16$, and $32 \mathrm{ps}$.

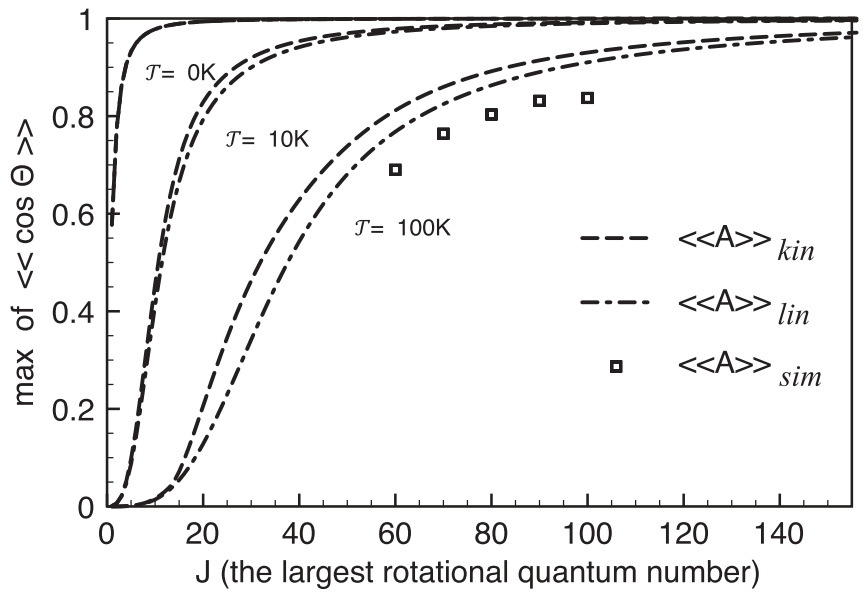

FIG. 2. Bounds of the OCS orientation as a function of $J$ at $\mathcal{T}=0,10$, and $100 \mathrm{~K}$. Kinematic bound $\left\langle\langle A\rangle_{\text {kin }}\right.$ : dashed curves (- - ); upper bound for linearly polarized fields $\langle\langle A\rangle\rangle_{\text {lin }}$ : dotted-dashed curves $(-\cdot-\cdot)$; numerically simulated bound $\left\langle\langle A\rangle_{\text {sim }}\right.$ : open squares $(\square)$ for $J=60,70,80,90,100$. Note that $\langle\langle A\rangle\rangle_{\text {kin }}=\langle\langle A\rangle\rangle_{\text {lin }}$ at $\mathcal{T}=0$ $\mathrm{K}$ (corresponding to the $m=0$ case), independent of $J$.

$B=6081.492 \mathrm{MHz}$, a centrifugal distortion constant $D=$ $1.301777 \mathrm{kHz}$ [39], and a permanent dipole $\mu_{0}=0.709 \mathrm{D}$ [40]. In general, the highest rotational quantum number $J$ in the simulations needs to be increased with the temperature, as well as with the intensity of the control field. To assess the maximum attainable field-free orientation of the OCS molecule with intense (as long as it is not too strong, e.g., $\leqslant 0.01$ a.u. [41]) short $\mathrm{THz}$ pulses, optimal control simulations for various OCS nonrigid rotor models are performed for different $J$ 's (here $J=60,70,80,90,100$, since in practice the simulation can only be performed at some finite $J$ values) according to the estimated initial state population distributions over the rotational energy levels $(j \leqslant J)$ at various temperatures (here $\mathcal{T}=0,10$, and $100 \mathrm{~K}$ ). The optimal control fields generated from the simulations at these finite $J$ 's are then used to compute

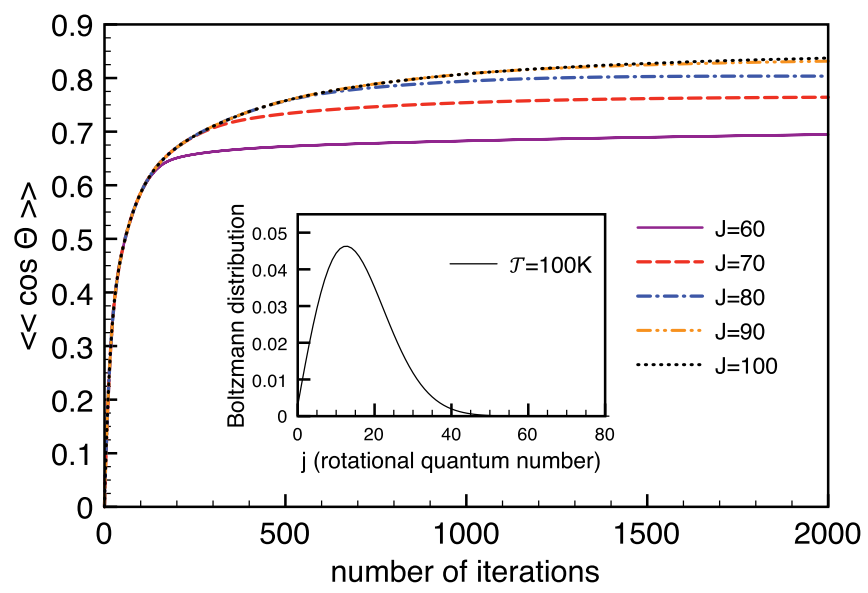

FIG. 3. (Color online) Optimal control simulations of the OCS orientation for $J=60,70,80,90,100$ at $\mathcal{T}=100 \mathrm{~K}$. The inset shows the Boltzmann distribution of the initial populations as a function of rotational quantum number $j$. 

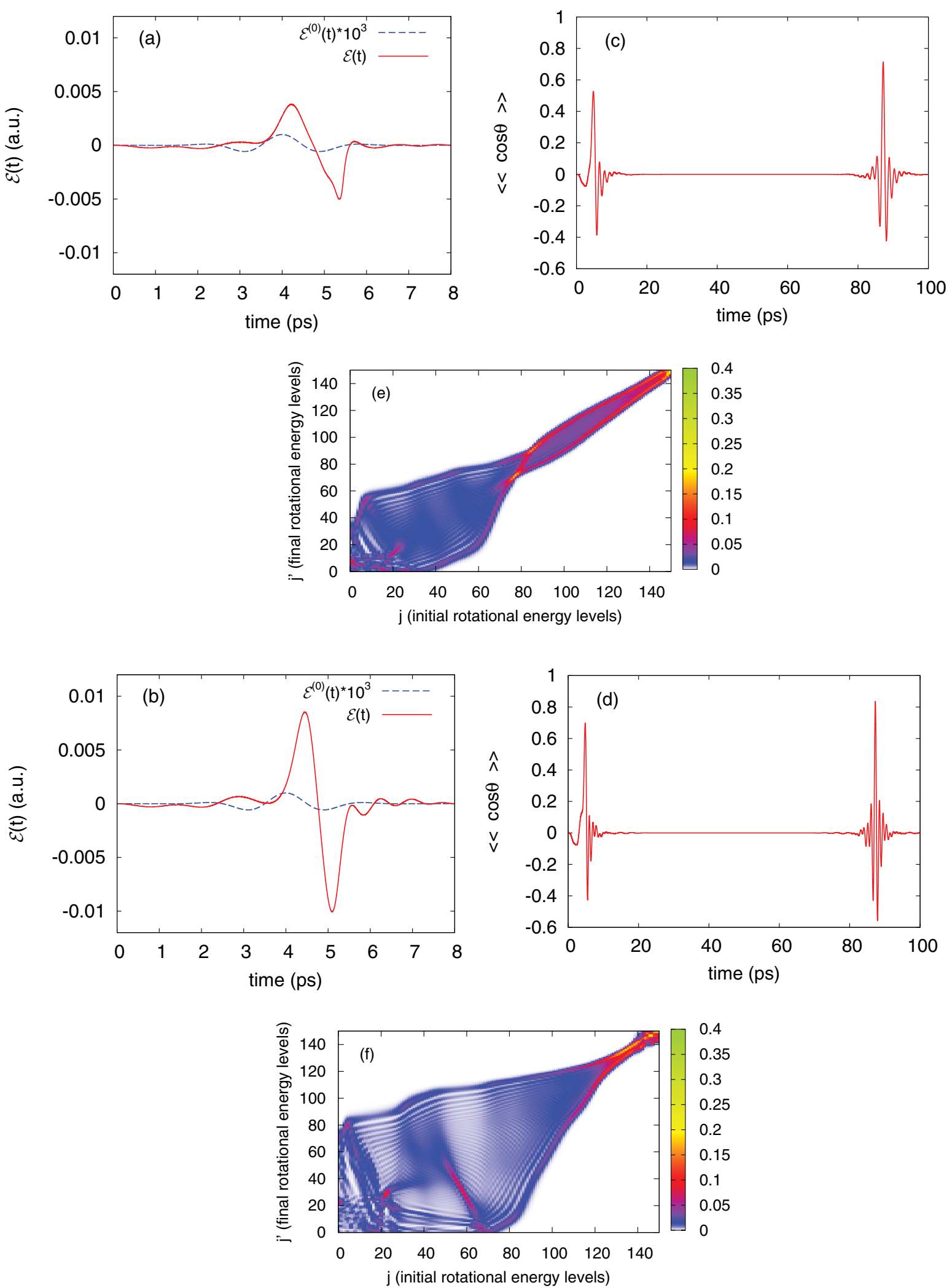

FIG. 4. (Color online) Optimal control results using the MS-TBQCP method: (a) and (b) are, respectively, optimal fields corresponding to $J=60$ and 100, and the dashed line is the initial control field $\mathcal{E}^{(0)}(t)$, which is a product of a Gaussian shape function (with a maximum amplitude equal to $10^{-6}$ a.u., a full width at half-maximum equal to $1.5 \mathrm{ps}$, and peaked at $t=4.0 \mathrm{ps)}$ and a cosine function cos $2 \pi f t$ (with a carrier frequency $f=0.5 \mathrm{THz}$ ); (c) and (d) are the time-dependent orientation of the OCS calculated corresponding to $J=150$ based on the optimal fields in (a) and (b), respectively; (e) and (f) are the contour plots of the transition probabilities $\left|c_{j^{\prime} m}^{j m}(T)\right|^{2}$, Eq. (9), between different initial and final rotational energy levels $j$ and $j^{\prime}$ (for $m=0$ ) at the target time $T \approx 87 \mathrm{ps}$ in (c) and (d), respectively. 
the field-field orientation at a much larger $J$ (here $J=150$ ) to show their validity.

In the calculations, we consider near-single-cycle linearly polarized $\mathrm{THz}$ control pulses. The pulse length of the control fields is fixed at $T_{1}=8 \mathrm{ps}$, which is less than one-tenth of the OCS rotational period, which is 82 ps. To this end, all control fields are written in a product form: $\mathcal{E}(t)=$ $S(t) F(t)$, where the shape function $S(t)$ is chosen to be of the form

$$
S(t)= \begin{cases}\sin ^{2}\left(\frac{\pi t}{T_{1}}\right) & \text { if } 0 \leqslant t \leqslant T_{1}, \\ 0 & \text { if } t<0 \text { or } t>T_{1},\end{cases}
$$

accounting for the near-single-cycle $\mathrm{THz}$ control pulses, and $F(t)$ is an arbitrary function of time $t$. The target time $T$ can be arbitrarily chosen as long as $T \geqslant T_{1}$ such that the maximum of the orientation can be obtained in the field-free regime. However, in all simulations presented in the paper, $T$ is chosen differently such that at each iteration $T$ is made to coincide with the instant when the degree of field-free orientation reaches the maximum at the previous iteration; thus $T$ converges as the final iteration is approached. For the OCS simulation, the ultimate maximum $\langle\langle A\rangle\rangle_{\text {sim }}$ of the field-free orientation occurs at a time nearly one rotational period after the pulse is terminated, i.e., $T \approx 87 \mathrm{ps}$. The maximum degree of orientation increases monotonically with the iteration.

The orientation process is in general controllable [28] when no constraints are placed on the controls. However, maximum orientation may still not be reached if the control pulse is too short (here $T_{1}=8 \mathrm{ps}$, which is much smaller than the rotational period of the OCS molecule at $82 \mathrm{ps}$ ). To this end, we perform the optimal orientation control simulations for the OCS molecule (with $J=60$ ) at the temperature $\mathcal{T}=0 \mathrm{~K}$ for pulses of length equal to $T_{1}=8,16$, and $32 \mathrm{ps}$ (no other constraints, including the frequency one, on the control pulses are imposed here). Figure 1 shows that the numerically simulated maximal orientation, $\langle\langle A\rangle\rangle_{\text {sim }}$, after 2000 iterations, by a very short linearly polarized pulse (here $T_{1}=8 \mathrm{ps}$ ), is less than the estimated upper bound $\langle\langle A\rangle\rangle_{\text {lin }}$ that can be readily achieved with a long linearly polarized pulse (here $T_{1}=32 \mathrm{ps}$ ).

Figure 2 presents the kinematic bound $\langle\langle A\rangle\rangle_{\text {kin }}$ (dashed curves) and the linear bound $\langle\langle A\rangle\rangle_{\text {lin }}$ (dotted-dashed curves) as a function of $J$ at $\mathcal{T}=0,10$, and $100 \mathrm{~K}$, and the numerically simulated bound $\langle\langle A\rangle\rangle_{\text {sim }}$ (open squares) for $J=60,70,80,90,100$ at $\mathcal{T}=100 \mathrm{~K}$. It is found that (i) the maximum attainable orientation increases as the number of rotational states increases; (ii) the kinematic bound $\langle\langle A\rangle\rangle_{\text {kin }}$ always lies above the optimal bound $\left\langle\langle A\rangle_{\text {lin }}\right.$; and (iii) the simulation maximal yields $\langle\langle A\rangle\rangle_{\text {sim }}$ at $\mathcal{T}=100 \mathrm{~K}$ are slightly smaller than their $\langle\langle A\rangle\rangle_{\text {lin }}$ counterparts. Figure 3 plots the simulation maximum orientation $\left\langle\langle A\rangle_{\text {sim }}\right.$, i.e., the maximum attainable average value of $\cos \theta$, as a function of iteration at $\mathcal{T}=100 \mathrm{~K}$ for various $J$ values $\left(\langle\langle A\rangle\rangle_{\text {sim }}=0.694\right.$ and 0.837 for $J=60$ and 100 , respectively, after 2000 iterations, compared to $\langle\langle A\rangle\rangle_{\text {lin }}=0.768$ for $J=60$ and 0.910 for $J=100$ ); the smooth convergent curves show the monotonic property of the MS-TBQCP algorithm. The inset shows the Boltzmann distribution of the initial populations at different rotational energy levels (i.e., as a function of rotational quantum number $j$ ). It is seen that only the rotational energy levels with $j \leqslant 50$ are significantly populated (i.e., $>10^{-3}$ ) at $\mathcal{T}=100 \mathrm{~K}$.

Figures 4(a) and 4(b) depict, respectively, the optimized laser pulses, as a function of $t$, for $J=60$ and 100 at $\mathcal{T}=$ 100 K. Plotted in Figs. 4(c) and 4(d) are the time-dependent orientation calculated for $J=150$ using the optimal fields generated for $J=60$ and 100 , respectively, showing that these optimally shaped fields based on lower $J$ 's can still render nearly maximum orientation attainable at a much larger $J$. Specifically, our simulations show that a larger $J$ (here $J=$ 100 compared to $J=60$ ) results in a stronger optimal control pulse (amplitude $\approx 0.005$ a.u. for $J=60$ and $\approx 0.01$ a.u. for $J=100)$ and a larger orientation (here $\langle\langle\cos \theta\rangle\rangle=0.714$ and 0.837 for $J=60$ and 100, respectively). Presented in Figs. 4(e) and 4(f) are the transition probabilities $\left|c_{j^{\prime} m}^{j m}(T)\right|^{2}$, Eq. (9), between different initial and final rotational energy levels $j$ and $j^{\prime}$ (within the $m=0$ subspace) at the target time $T$, showing that the resultant intense near-single-cycle $\mathrm{THz}$ pulses arising from both $J=60$ and 100 simulations are able to excite a large number of rotational levels coherently to form a well-oriented wave packet at the target time.

\section{SUMMARY}

We have presented a detailed analysis of the maximum attainable field-free orientation of OCS molecules (treated as nonrigid rotors) with near-single-cycle $\mathrm{THz}$ laser pulses at finite temperatures. To perform the needed large-scale benchmark model simulations, including rotational states with the rotational quantum numbers up to $J=100$, an efficient monotonically convergent algorithm for the mixed-states quantum optimal controls has been implemented, based on a recently developed TBQCP method [31-33], to identify the desired optimal control pulses. It was shown from our large-scale $a b$ initio optimal control simulations that a nearly maximal degree of field-free orientation of OCS molecules could be achieved by strong, optimally shaped near-singlecycle $\mathrm{THz}$ pulses, in agreement with the basic understanding of how the field-free orientation is created through the nonadiabatic process. The extensive numerical simulations performed at $\mathcal{T}=100 \mathrm{~K}$ showed that the maximum attainable $J$-dependent orientation in the near-single-cycle $\mathrm{THz}$ pulse region is close to $92 \%$ of the corresponding optimal bounds that can be attained by arbitrarily long pulses. It is also found that a smaller amplitude for the optimal control field corresponds to a smaller $J$. This finding may underline the actual experimental performance of the field-free molecular orientation, since presently the amplitude of single-cycle $\mathrm{THz}$ pulses can only reach slightly beyond $20 \mathrm{MV} / \mathrm{cm}(\approx 0.0038$ a.u. $)$ in the laboratory [34].

\section{ACKNOWLEDGMENTS}

This work was partially supported by the US Department of Energy. We would like also to acknowledge the partial support of the National Science Council of Taiwan and National Taiwan University (Grants No. 102R104021, No. 102R8700-2). 
[1] M. Y. Ivanov and J. P. Marangos, J. Mod. Opt. 54, 899 (2007).

[2] J. Itatani, J. Levesque, D. Zeidler, H. Niikura, H. Pepin, J. C. Kieffer, P. B. Corkum, and D. M. Villeneuve, Nature (London) 432, 867 (2004).

[3] M. Meckel, D. Comtois, D. Zeidler, A. Staudte, D. Pavicic, H. C. Bandulet, H. Pepin, J. C. Kieffer, R. Dorner, D. M. Villeneuve, and P. B. Corkum, Science 320, 1478 (2008).

[4] I. V. Litvinyuk, K. F. Lee, P. W. Dooley, D. M. Rayner, D. M. Villeneuve, and P. B. Corkum, Phys. Rev. Lett. 90, 233003 (2003).

[5] T. Suzuki, S. Minemoto, T. Kanai, and H. Sakai, Phys. Rev. Lett. 92, 133005 (2004).

[6] T. Kanai, S. Minemoto, and H. Sakai, Nature (London) 435, 470 (2005).

[7] T. Kanai, S. Minemoto, and H. Sakai, Phys. Rev. Lett. 98, 053002 (2007).

[8] H. Stapelfeldt and T. Seideman, Rev. Mod. Phys. 75, 543 (2003).

[9] T. Seideman and E. Hamilton, Adv. At. Mol. Opt. Phys. 52, 289 (2005).

[10] A. Pelzer, S. Ramakrishna, and T. Seideman, J. Chem. Phys. 126, 034503 (2007).

[11] B. Friedrich and D. R. Herschbach, Nature (London) 353, 412 (1991).

[12] B. Friedrich and D. Herschbach, J. Chem. Phys. 111, 6157 (1999).

[13] T. Kanai and H. Sakai, J. Chem. Phys. 115, 5492 (2001).

[14] S. De, I. Znakovskaya, D. Ray, F. Anis, N. G. Johnson, I. A. Bocharova, M. Magrakvelidze, B. D. Esry, C. L. Cocke, I. V. Litvinyuk, and M. F. Kling, Phys. Rev. Lett. 103, 153002 (2009).

[15] R. Tehini and D. Sugny, Phys. Rev. A 77, 023407 (2008).

[16] M. Machholm and N. E. Henriksen, Phys. Rev. Lett. 87, 193001 (2001).

[17] C. Dion, A. Keller, and O. Atabek, Eur. Phys. J. D 14, 249 (2001).

[18] C.-C. Shu, K.-J. Yuan, W.-H. Hu, and S.-L. Cong, Phys. Rev. A 80, 011401 (2009).

[19] S. Fleischer, Y. Zhou, R. W. Field, and K. A. Nelson, Phys. Rev. Lett. 107, 163603 (2011).

[20] A. Ben Haj-Yedder, A. Auger, C. M. Dion, E. Cancès, A. Keller, C. Le Bris, and O. Atabek, Phys. Rev. A 66, 063401 (2002).
[21] B. J. Sussman, J. G. Underwood, R. Lausten, M. Y. Ivanov, and A. Stolow, Phys. Rev. A 73, 053403 (2006).

[22] M. Muramatsu, M. Hita, S. Minemoto, and H. Sakai, Phys. Rev. A 79, 011403 (2009)

[23] C.-C. Shu, K.-J. Yuan, W.-H. Hu, and S.-L. Cong, J. Chem. Phys. 132, 244311 (2010).

[24] S. Zhang, J. Shi, H. Zhang, T. Jia, Z. Wang, and Z. Sun, Phys. Rev. A 83, 023416 (2011).

[25] C. M. Dion, A. B. Haj-Yedder, E. Cancès, C. Le Bris, A. Keller, and O. Atabek, Phys. Rev. A 65, 063408 (2002).

[26] D. Sugny, A. Keller, O. Atabek, D. Daems, C. M. Dion, S. Guerin, and H. R. Jauslin, Phys. Rev. A 69, 033402 (2004).

[27] J. Salomon, C. M. Dion, and G. Turinici, J. Chem. Phys. 123, 144310 (2005).

[28] D. Sugny, A. Keller, O. Atabek, D. Daems, C. M. Dion, S. Guerin, and H. R. Jauslin, Phys. Rev. A 72, 032704 (2005).

[29] Y. Ohtsuki and K. Nakagami, Phys. Rev. A 77, 033414 (2008).

[30] M. Lapert, R. Tehini, G. Turinici, and D. Sugny, Phys. Rev. A 78, 023408 (2008).

[31] T.-S. Ho and H. Rabitz, Phys. Rev. E 82, 026703 (2010).

[32] T.-S. Ho, H. Rabitz, and S.-I. Chu, Comput. Phys. Commun. 182, 14 (2011)

[33] S.-L. Liao, T.-S. Ho, S.-I. Chu, and H. Rabitz, Phys. Rev. A 84, 031401(R) (2011).

[34] D. Daranciang, J. Goodfellow, M. Fuchs, H. Wen, S. Ghimire, D. A. Reis, H. Loos, A. S. Fisher, and A. M. Lindenberg, Appl. Phys. Lett. 99, 141117 (2011).

[35] E. Hamilton, T. Seideman, T. Ejdrup, M. D. Poulsen, C. Z. Bisgaard, S. S. Viftrup, and H. Stapelfeldt, Phys. Rev. A 72, 043402 (2005).

[36] J. Stoustrup, O. Schedletzky, S. J. Glaser, C. Griesinger, N. C. Nielsen, and O. W. Sørensen, Phys. Rev. Lett. 74, 2921 (1995).

[37] S. G. Schirmer and J. V. Leahy, Phys. Rev. A 63, 025403 (2001)

[38] T.-S. Ho and H. Rabitz, J. Photochem. Photobiol., A 180, 226 (2006).

[39] A. G. Maki, J. Phys. Chem. Ref. Data 3, 221 (1974).

[40] R. G. Shulman and C. H. Townes, Phys. Rev. 77, 500 (1950).

[41] J. H. Nielsen, H. Stapelfeldt, J. Küpper, B. Friedrich, J. J. Omiste, and R. González-Férez, Phys. Rev. Lett. 108, 193001 (2012). 